\title{
Alteración de la velocidad de la marcha y del test de levantarse de la silla: ¿lnicio del síndrome de fragilidad en mujeres mayores institucionalizadas?
}

\author{
Ruiz-Rivera Raymundo*, Cruz-Castruita Rosa María*, Morales-Elizondo Dulce Edith*, \\ Ramírez Orozco Ricardo Ernesto**
}

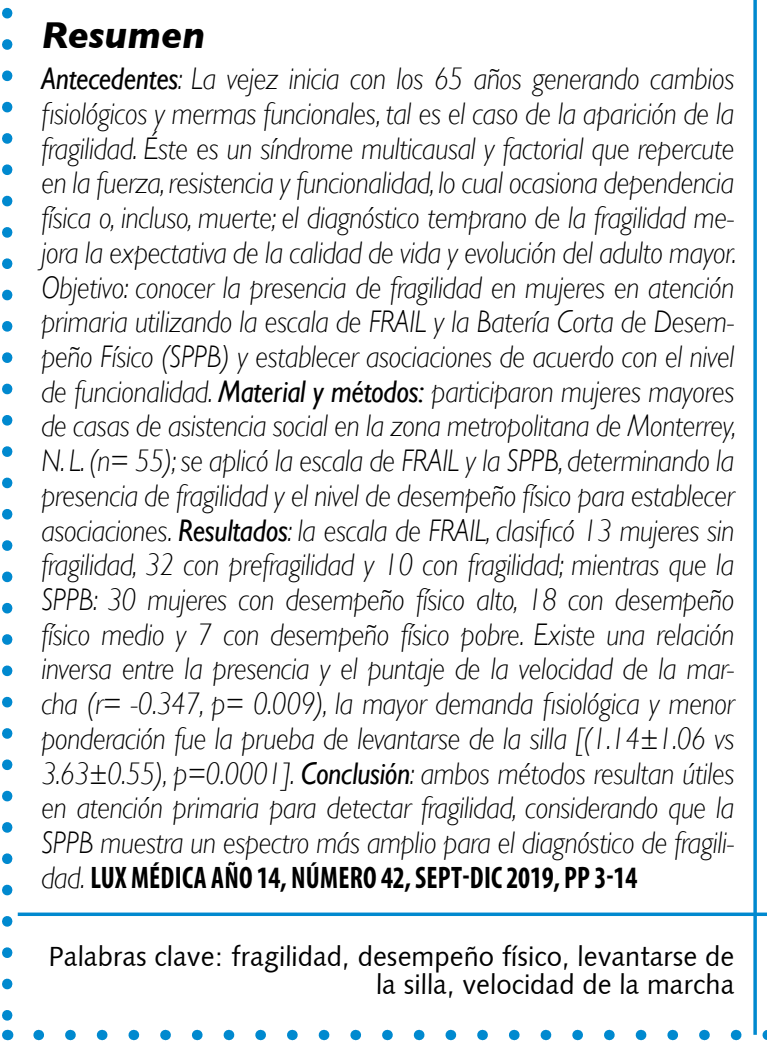

\section{Abstract}

Introduction: Old age begins at 65 years, generating physiological changes and functional losses, such as frailty. Frailty is a multicausal and factorial syndrome that affects strength, endurance and functionality, causing physical dependence or even death. The early diagnosis of frailty creates an expectation of the quality of life and evolution of the elderly. Objective: To recognize the presence of frailty in women in primary care using the FRAll scale and the Short Physical Performance Battery (SPPB) and to establish associations according to the level of functionality. Methods: Participants were elderly women $(n=55)$ from social welfare homes in the metropolitan area of Monterrey, N. L; the FRAIL scale and the SPPB were applied, determining the presence of frailty and the level of physical performance to establish associations. Results: The FRAll scale classified 13 women without frailty, 32 with pre-frailty and, 10 with frailty. While the SPPB: 30 women with high physical performance, 18 with medium physical performance and, 7 with poor physical performance. There is an inverse relationship between the presence and the gait speed score $(r=-0.347, p=0.009)$, the higher physiological demand and lower weighting was the chair rise test $[(1.14 \pm 1.06$ vs. $3.63 \pm 0.55), p$ $=0.000$ I]. Conclusions: Both methods are useful in primary care to detect frailty, considering that the SPPB shows a broader spectrum for the diagnosis of fragility. LUX MÉDICA AÑO 14, NÚMERO 42, SEPT-DIC 2019, PP 3-14

Keywords: frailty, physical performance, chair rise, gait speed

Raymundo Ruiz Rivera, Facultad de Organización Deportiva. Universidad Autónoma de Nuevo León, Av. Pedro de Alba S/N, Ciudad Universitaria. San Nicolás de los Garza, Nuevo León, México, C.P. 6645. Correo electrónico rayruiz1224@gmail.com

Rosa María Cruz Castruita. Universidad Autónoma de Nuevo León. Departamento Subdirección del área de posgrado. Correo electrónico castruita_81@hotmail.com

* Dulce Edith Morales Elizondo. Universidad Autónoma de Nuevo León. Promoción de la Salud. Correo electrónico: dulce.moraleslz@uanledu.mx

** Profesor Investigador del Departamento de Nutrición del Centro de Ciencias de la Salud de la Universidad Autónoma de Aguascalientes. Correo electrónico: dcmrero@gmail.com

Fecha de recibido: 26 de marzo 2019

Fecha de aceptación: 1 de agosto 2019

Correspondencia: Dr. en C Ricardo Ernesto Ramírez Orozco. Universidad Autónoma de Aguascalientes, Departamento de Nutrición. Avenida Universidad \#940, Ciudad Universitaria Código Postal 20131 Aguascalientes, Ags., México. 9107400 ext. 53355 Correo electrónico: dcmrero@gmail.com 


\section{Introducción}

Hoy en día el crecimiento demográfico ha incrementado considerablemente la población de adultos mayores superando la esperanza de vida los 70 años. En los próximos 50 años se espera que la población de adultos mayores pase de 600 a 2000 millones. ${ }^{1}$ En el año 2013 se estimó una esperanza de vida en población mexicana de 75.4 años, mientras que la expectativa de una salud adecuada es de 65.8 años. La presencia de discapacidad y otros factores como enfermedades afectan a los adultos mayores durante los últimos diez años de vida, aquejando mayormente a hombres. ${ }^{2,3}$

Durante el proceso de envejecimiento, el adulto mayor se ve expuesto al desarrollo de enfermedades y problemas geriátricos. ${ }^{4}$ La fragilidad es un síndrome clínico de causas y agentes múltiples, que se caracteriza por la pérdida de la fuerza y/o resistencia y disminución de la función fisiológica, que aumenta la vulnerabilidad individual para desarrollar dependencia física o incluso la muerte. ${ }^{5}$ Aproximadamente el 10\% de los adultos mayores de 65 años son frágiles, prevalencia que aumenta hasta $75 \%$ en personas mayores de 85 años. La presencia de fragilidad condiciona un incremento en el riesgo de caídas, fracturas, ingresos hospitalarios, deterioro funcional, e institucionalización en asilos. ${ }^{6}$

Dentro de las opciones terapéuticas del síndrome de fragilidad, el ejercicio físico constituye un punto clave, ya que revierte la discapacidad y factores adversos que se asocian al síndrome. El entrenamiento denominado multicomponente es considerado el más eficaz contra la fragilidad, debido a la combinación de entrenamientos de fuerza, resistencia, equilibrio y flexibilidad. ${ }^{7}$ Por otro lado, el síndrome de fragilidad está asociado al sexo femenino, presencia de enfermedades, deterioro cognitivo, aunado a factores genéticos, hormonales, procesos de inflamación, estrés oxidativo, neuromusculares y nutricionales. ${ }^{8}$ No se ha logrado establecer un estándar de oro para el diagnóstico de fragilidad; sin embargo, se han considerado y desarrollado instrumentos fiables y consensuados que permiten relacionar la presencia de este síndrome. ${ }^{8}$ La fragilidad ha sido estudiada desde diversos ángulos con la intención de generar un diagnóstico preciso, entre los cuales se pueden destacar: el desempeño físico, indicadores de fatiga, discapacidad, o presencia de comorbilidades. ${ }^{9}$

Entre los constructos y escalas más utilizadas para su diagnóstico, encontramos el fenotipo de fragilidad, ${ }^{10}$ que considera una perspectiva biológica, la acumulación de déficits ${ }^{11}$ y finalmente la escala de FRAIL. ${ }^{12}$ La evolución funcional es un factor importante dentro del proceso diagnóstico y terapéutico del adulto mayor, considerando que el deterioro funcional es un fuerte predictor de la mala evolución clínica y de mortalidad en adultos mayores. ${ }^{13,14}$ La SPPB (Batería Corta de Desempeño Físico) evalúa el desempeño físico en adultos mayores, además de ser una herramienta práctica $y$ eficiente ${ }^{15}$ que predice el deterioro en la funcionalidad, siendo 
utilizada como auxiliar en el diagnóstico de fragilidad. ${ }^{16-18}$ La detección de fragilidad y deterioro del desempeño físico en adultos mayores es fundamental en la atención geriátrica, ya que permite la prevención y aplicación de nuevos programas de intervención. El objetivo de este estudio fue la detección de fragilidad y la evaluación del desempeño físico en mujeres adultas mayores que se encuentran institucionalizadas en la ciudad de Monterrey, Nuevo León, México.

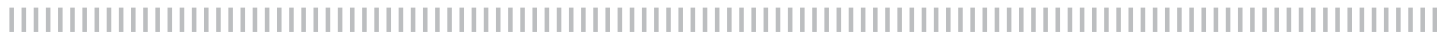

\section{Material y Métodos}

El diseño del estudio fue de tipo descriptivo, correlacional y de corte transversal. La muestra del estudio fue conformada por mujeres adultas mayores $(n=55)$ con una edad entre los 60 y 94 años de acuerdo con los criterios de la Organización Mundial de la Salud (OMS). Las participantes fueron mujeres inscritas a un padrón de asistencia de alguna de las casas club de asistencia social (Casa club Los altos y Casa club Lomas Modelo) para adultos mayores del Sistema para el Desarrollo Integral de la Familia (DIF) de la zona metropolitana de Monterrey, Nuevo León, México. Se generó un muestreo no probabilístico y por conveniencia, con la participación voluntaria en el estudio, además del consentimiento informado. Se excluyeron personas con dispositivos cardiacos como marcapasos; se aplicó la misma indicación en personas con problemas de comunicación y/o personas que presentaran alguna contraindicación médica para realizar actividad física o alguna de las pruebas que componen la SPPB.

Evaluación de la fragilidad: se utilizó la escala de FRAIL de fácil aplicación, compuesta por 5 ítems (fatiga, resistencia, deambulación, comorbilidad y pérdida de peso). Cada respuesta afirmativa es valorada con 1 punto, se considera la presencia de fragilidad con una puntuación $\geq$
3 y pre-fragilidad con $1 \circ 2 .{ }^{19}$ La batería cuenta con el antecedente de haber sido validada en población mexicana. ${ }^{20}$

Evaluación del desempeño físico: fue evaluado por la Batería Corta de Desempeño Físico (SPPB), que se compone de tres pruebas: equilibrio, velocidad de la marcha y fuerza para levantarse de una silla; ${ }^{21}$ a cada parámetro de la batería se le asigna una puntuación en una escala de 0 a 4 . La puntuación global de la batería va de 0 a 12 puntos; de 0 a 6 puntos se clasifica como desempeño físico bajo; de 7 a 9 , como desempeño físico intermedio; y de 10 a 12, como desempeño físico alto. ${ }^{22}$

Análisis estadístico: los datos fueron representados como: media, desviación estándar o mediana y rangos intercuartiles $(25 \%-75 \%)$, según lo apropiado. La normalidad se evaluó con el test de Kolmogorov-Smirnov. Se aplicó un ANOVA no paramétrico (Kruskal-Wallis) y el post hoc de Dunn's, para buscar diferencias entre niveles de desempeño o presencia de fragilidad. Se utilizó el coeficiente de correlación de Pearson para establecer asociaciones entre los puntajes de las pruebas de fragilidad, desempeño físico y variables antropométricas; los datos fueron evaluados mediante el paquete estadístico SPSS versión. ${ }^{23}$ Se consideró una $p<0.05$ estadísticamente significativa.

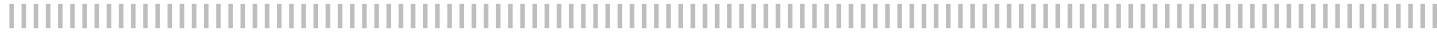

\section{Resultados}

1. Características descriptivas de la población estudiada.
Las mujeres adultas mayores que participaron en el estudio $(n=55)$, reportaron una edad entre los 60 y 94 años con una 
media de $72 \pm 7.2$ años. Las características sociodemográficas como: estado civil, vivienda, rol laboral y dependencia económica se representan en la tabla 1. El $43.6 \%$ $(n=24)$ de las mujeres vivía en viudez; el $41.8 \%(n=23)$, con su pareja; y el restante, con algún hijo. En relación con las actividades laborales, el $45.5 \%(n=25)$ recibe una pensión; y el $36.4 \% \quad(n=20)$ de ellas dijo dedicarse a realizar actividades domésticas.

En cuanto a las variables antropométricas y clínicas, las mujeres adultas mostraron bajo peso en el 5.5\% $(n=5) ; 34.5 \%$ $(n=19)$, un peso normal; $30.9 \%(n=19)$, sobrepeso; y $29.1 \% \quad(n=16)$, obesidad. Variables como el consumo de medicamentos, el $12.7 \% \quad(n=7)$ consumen de 4 a 5 medicamentos y $7.3 \% \quad(n=4)$ más de 5. Finalmente, resultados obtenidos por el cuestionario FRAIL, diagnosticaron $10(18.18 \%)$ mujeres con fragilidad, 32 $(58.18 \%)$ con prefragilidad y 13 sin fragilidad 13 (23.63\%). De estas mujeres, $7(12.72 \%)$ tienen un desempeño físico bajo; 18 (32.72\%), un desempeño físico intermedio; y 30 (54.54\%), un nivel de desempeño alto. En la tabla 1 se exponen el resto de las características estudiadas.

\section{Tabla I}

\section{Características socio demográficas de la población de adultas mayores}

\begin{tabular}{|llllll|}
\hline Características & $\mathrm{f}$ & $\%$ & Características & $\mathrm{f}$ & $\%$ \\
\hline Estado Civil & & & En qué trabaja & \\
\hline Casado & 23 & 41.8 & Hogar & 20 & 36.4 \\
\hline Viudo & 24 & 43.6 & Pensionado & 25 & 45.5 \\
\hline Divorciado & 10 & 5.5 & Jubilado & 2 & 3.6 \\
\hline Vive solo & & & Otro & 8 & 14.5 \\
\hline Sí & 14 & 25.5 & Dependencia económica & & \\
\hline No & 41 & 74.5 & Sí & 32 & 58.2 \\
\hline Con quién vive & & & No & 23 & 41.8 \\
\hline Solo & 13 & 23.6 & De quién & 22 & 40 \\
\hline Esposo & 23 & 41.8 & De nadie & 19 & 34.5 \\
\hline Hijo (a) & 15 & 27.3 & Esposo & 14 & 25.5 \\
\hline Hermano (a) & 2 & 3.6 & Hijo (a) & \\
\hline Nieto (a) & 2 & 3.6 & & \\
\hline
\end{tabular}

Datos representados como frecuencias y porcentajes $(n=55) ; f=$ frecuencia; $\%=$ porcentaje.

2. Desempeño físico bajo y su influencia en la merma de equilibrio

Por un lado, en las mujeres evaluadas se pudo observar el desempeño físico como una determinante en la prueba de equilibrio $[(2.43 \pm 1.39$ vs $3.83 \pm 0.37)$, $p=0.0001]$, es decir, al menos un desempeño intermedio será necesario para mantener un equilibrio físico adecuado. Por otro, el test de velocidad de la marcha [(1.86 \pm 0.69 vs $3.60 \pm 0.49), p=0.0001] y$ el test de levantarse de la silla [(1.14 1.06 vs $3.63 \pm 0.55), p=0.0001]$, requieren mayor destreza y por lo tanto, desempeño para efectuarlos de manera correcta (Tabla 2 y Figura 1). 


\section{Tabla 2}

Diferencias entre las pruebas de SPPB y el nivel de desempeño físico en mujeres adultas mayores

\begin{tabular}{|lccccc|}
\hline Batería SPPB & DF. Bajo & DF. Intermedio & DF. Alto & $p$ & Post Hoc \\
\hline & $(\mathrm{n}=7)$ & $(\mathrm{n}=18)$ & $(\mathrm{n}=30)$ & & \\
\hline Test de equilibrio (puntos) & $2.43 \pm 1.39$ & $3.50 \pm 0.61$ & $3.83 \pm 0.37$ & 0.0001 & $0.0001^{\mathrm{a}}$ \\
\hline Test de la velocidad de la marcha (puntos) & $1.86 \pm 0.69$ & $2.56 \pm 1.04$ & $3.60 \pm 0.49$ & 0.0001 & $0.0001^{\mathrm{b}}$ \\
\hline Test de levantarse de la silla (puntos) & $1.14 \pm 1.06$ & $1.89 \pm 0.90$ & $3.63 \pm 0.55$ & 0.0001 & $0.0001^{\mathrm{b}}$ \\
\hline
\end{tabular}

Datos representados en medias y desviación estándar $(n=55) ; D F$ : Desempeño físico. a Diferencia encontradas entre el grupo de bajo desempeño físico contra los demás.

b Diferencia encontradas entre el grupo de alto desempeño físico contra los demás.
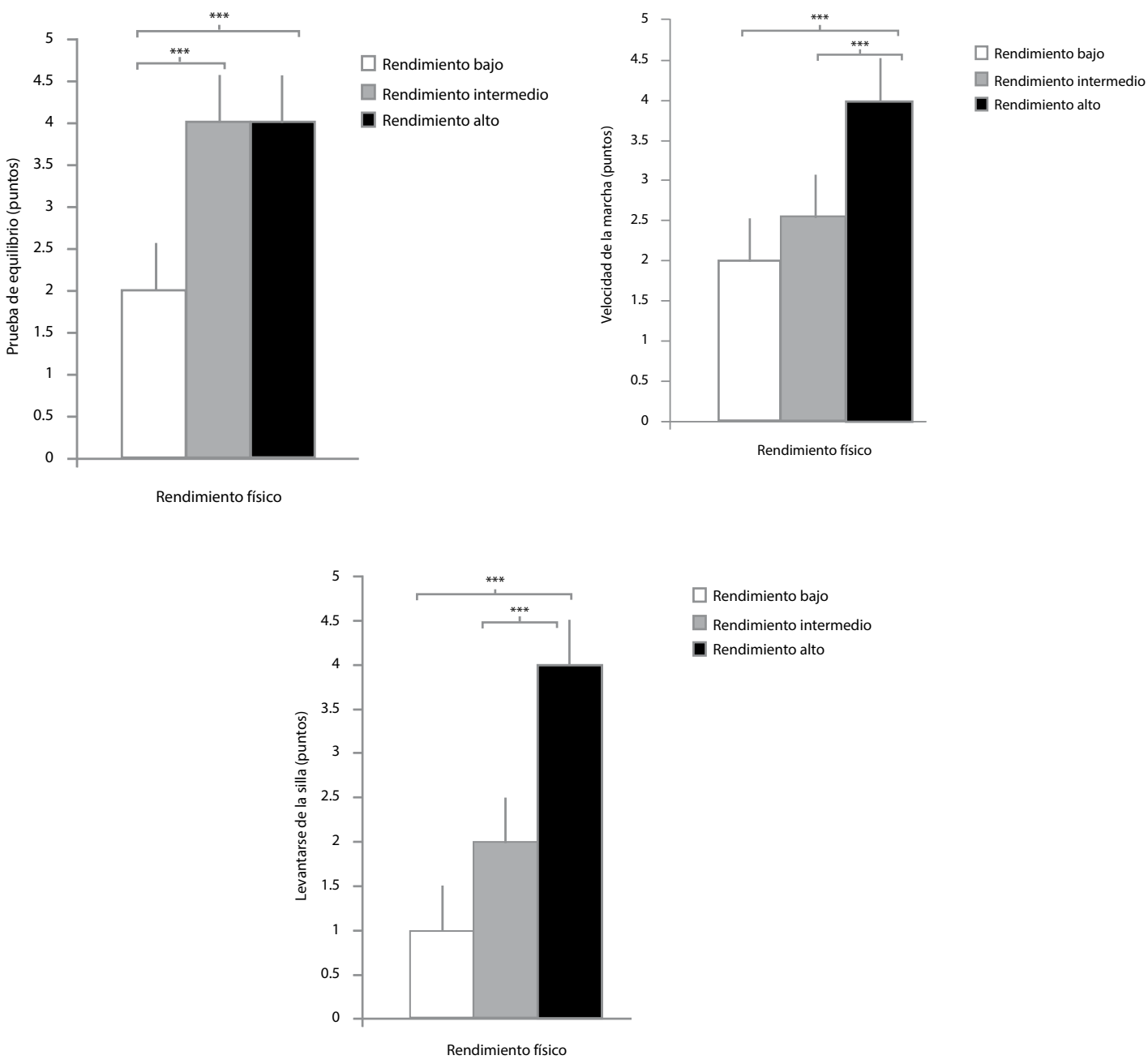

Figura 1. Evaluación del desempeño físico de acuerdo con la aplicación de la batería SPPB en mujeres adultas mayores. Pruebas de equilibrio (superior izquierda), velocidad de la marcha (superior derecha) y levantarse de la silla (inferior). ***: $p<0.001 ;(n=55)$. 
3. Influencia del desempeño físico en el desarrollo de fragilidad

En la prueba de velocidad de la marcha [(3.46 \pm 0.96 vs $2.50 \pm 0.85), p=0.029]$ y de levantarse la silla $[(2.46 \pm 1.33$ vs $1.90 \pm 0.87), p=0.006$ ] se puede observar un mejor puntaje en la prueba SPPB en el grupo de mujeres sin fragilidad en comparación con aquellas mujeres que desarrollan fragilidad presentando un puntaje pobre en las pruebas de rendimiento físico de la batería SPPB (Tabla 3 y Figura 2).

\section{Tabla 3}

\section{Diferencias entre SPPB y la presencia de fragilidad de mujeres adultas mayores}

\begin{tabular}{|lccccc|}
\hline Variables & Sin fragilidad & Prefragilidad & Fragilidad & $p$ & Post Hoc \\
\hline & $(\mathrm{n}=13)$ & $(\mathrm{n}=32)$ & $(\mathrm{n}=10)$ & & \\
\hline Test de equilibrio (puntos) & $4 \pm 1.05$ & $4 \pm 0.62$ & $4 \pm 0.25$ & 0.232 & 0.303 \\
\hline Test de la velocidad de la marcha (puntos) & $3.46 \pm 0.96$ & $3.03 \pm 0.96$ & $2.50 \pm 0.85$ & 0.029 & $0.029^{\mathrm{a}}$ \\
\hline Test de levantarse de la silla (puntos) & $2.46 \pm 1.33$ & $3.13 \pm 1.18$ & $1.90 \pm 0.87$ & 0.006 & $0.006^{\mathrm{b}}$ \\
\hline Batería SPPB (puntaje final) & $9.54 \pm 1.66$ & $9.78 \pm 2.16$ & $7.60 \pm 2.17$ & 0.026 & $0.026^{\mathrm{b}}$ \\
\hline
\end{tabular}

Datos representados en medias y desviación estándar. $(n=55)$.

a Diferencia encontradas entre el grupo de fragilidad en comparación al grupo sin fragilidad. b Diferencia encontradas entre el grupo de fragilidad en comparación al grupo con prefragilidad.
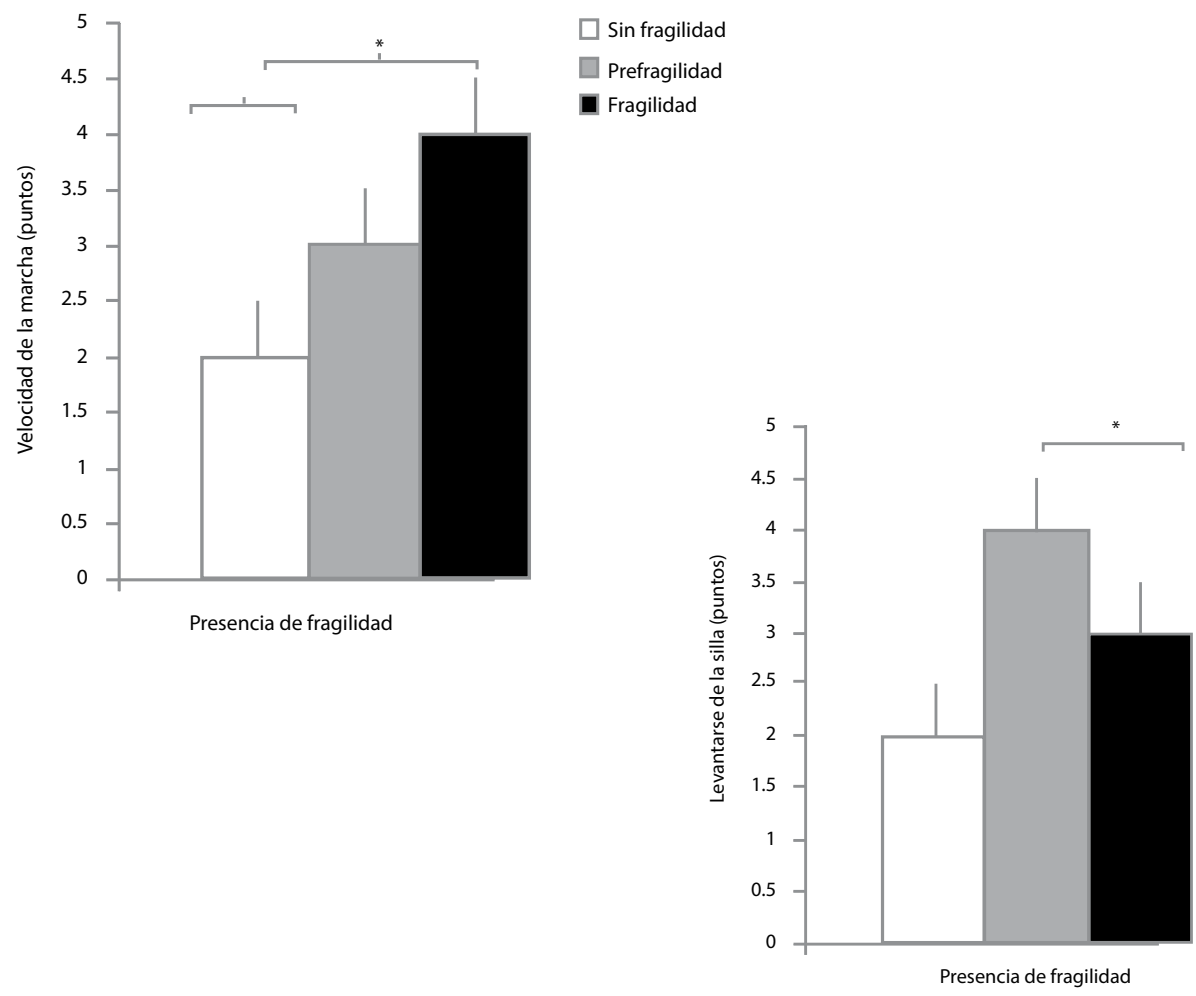

$\square$ Sin fragilidad 


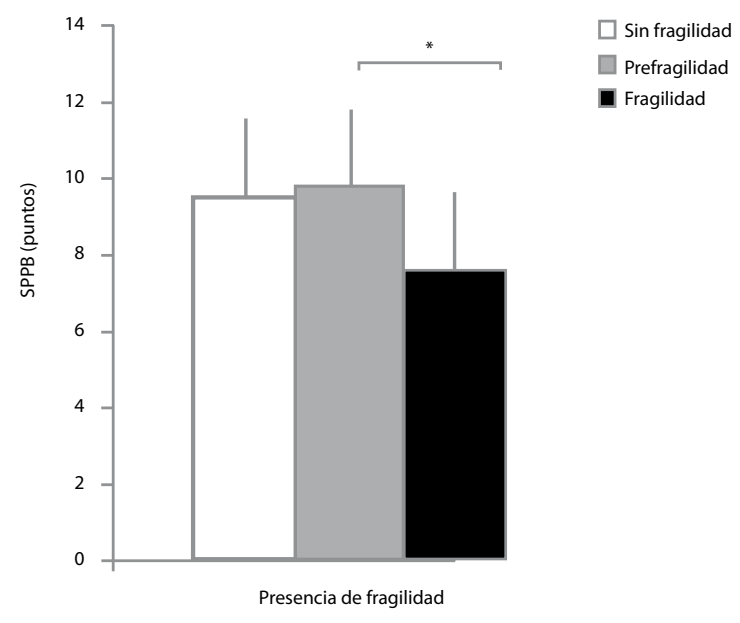

Figura 2. Evaluación del desempeño físico y su influencia en la presencia de fragilidad en mujeres adultas mayores. Pruebas de velocidad de la marcha (superior izquierda), levantarse de la silla (superior derecha) y puntaje final de la batería SPPB en conjunto (inferior). *: $p<0.05 ;(n=55)$.

4. Asociación entre la fragilidad y el desempeño físico en mujeres adultas mayores

La presencia de fragilidad y su relación con el nivel de desempeño físico, se estableció una relación inversa entre la velocidad de la marcha y la presencia de fragilidad ( $r=-0.347, p=0.009$ ), así como en el conjunto de tareas a realizar para evaluar el desempeño físico y su relación con la fragilidad ( $r=-0.324, p=0.016)$; es decir, un incremento en el puntaje del cuestionario FRAIL determinará una menor puntuación en la batería SPPB para evaluar el desempeño físico (Tabla 4).

\section{Tabla 4}

Relación entre la presencia de fragilidad y el nivel de desempeño físico.

\begin{tabular}{|llll|}
\hline Variables & Cuestionario FRAIL & r & P \\
\hline Batería SPPB (Puntaje final) & Cuestionario FRAIL (Puntos) & $\mathbf{- 0 . 3 2 4}$ & $\mathbf{0 . 0 1 6}$ \\
\hline Test de Equilibrio (Puntos) & Cuestionario FRAIL (Puntos) & -0.142 & 0.299 \\
\hline Test de Velocidad de la marcha (Puntos) & Cuestionario FRAIL (Puntos) & $-\mathbf{0 . 3 4 7}$ & $\mathbf{0 . 0 0 9}$ \\
\hline Test de Levantarse de la silla (Puntos) & Cuestionario FRAIL (Puntos) & -0.204 & 0.135 \\
\hline
\end{tabular}

Prueba estadística: Correlación de Pearson, $(n=55)$, se consideró estadísticamente significativa una p < 0.05 .

\section{Discusión}

La presencia de fragilidad y su asociación con la presencia y desarrollo de comorbilidades en el adulto mayor es una determi- nante en la calidad y desenlace que tendrá el adulto mayor en la última etapa de su vida; dentro de nuestro estudio, un cuarto de la población con fragilidad no presen- 
ta comorbilidades, es decir, ciertamente poseer padecimientos en la adultez mayor es una condicionante para desarrollar fragilidad; sin embargo, estudios similares de Soler y colaboradores muestran resultados semejantes, en donde el $26 \%$ de los adultos mayores, pese a no padecer enfermedades crónico degenerativas, desarrolla fragilidad. ${ }^{23,24}$ Esto podría ser explicado, probablemente, por el estado de funcionalidad previo a la discapacidad y dependencia, ya que es uno de los mejores indicadores y predictores del estado de salud y de discapacidad excluyente de la morbilidad. ${ }^{25}$ Un acercamiento a ello es el concepto de fragilidad, explicado como un estado de prediscapacidad, con riesgo a establecer una limitación funcional incipiente, centrando su importancia en la funcionalidad sin focalizar la presencia de enfermedad en el diagnóstico. ${ }^{26}$ Como parte del envejecimiento, personas mayores que se encuentran en la séptima década, presentan una disminución en su función fisiológica respecto a personas jóvenes; ${ }^{27,28}$ es decir, se ha observado una pérdida del $30-50 \%$ de neuronas motoras de los músculos de las piernas en adultos mayores sanos respecto a las que presentan personas jóvenes; esto sugiere una remodelación de la unidad motora, como parte de un proceso normal de envejecimiento. Esta pérdida no puede ser repuesta y conlleva a una declinación de los sistemas fisiológicos sin importar el nivel de actividad física, ${ }^{29}$ lo cual puede explicar parte de los resultados anteriormente mencionados.

La prevalencia de prefragilidad $(58.18 \%)$ y fragilidad $(18.18 \%)$ reportada en nuestro estudio es similar a la descrita por Huyen Thi Thahn y colaboradores, en su estudio en Vietnam con pacientes hospitalizados, que reportaron una tasa conjunta entre prefragilidad y fragilidad del $75.5 \%$, con $35.4 \%$ pacientes con fragilidad usando la escala de Fried y $31.9 \%$ con la Escala de Fragilidad de Edmonton (REFS). ${ }^{30}$ Los datos son similares a los obtenidos; sin embargo, la población de nuestro estudio presentó una tasa elevada de este padecimiento, aun sin ser pacientes hospitalizados, probablemente debido a los antecedentes de malnutrición que encontramos en nuestros participantes, el $75.5 \%$ de ellos presenta bajo peso, sobrepeso u obesidad. La malnutrición juega un papel crucial en el desarrollo de la fragilidad por medio de la sarcopenia: sin importar si es por desnutrición u obesidad, ambos incrementan el riesgo de fragilidad en población adulta mayor, por lo que la calidad de la dieta tiene una asociación directa con la incidencia de nuevos casos de este síndrome. ${ }^{31-33}$

La capacidad funcional es una característica conformada por múltiples factores, entre los cuales se incluye el desempeño físico con variables como fuerza, agarre, equilibrio, velocidad, potencia y algunos autores consideran importante la inclusión del nivel de conciencia, humor y variables de salud mental; en el presente estudio, al aplicar la SPPB para evaluar el desempeño físico, el $45 \%$ presentó un desempeño pobre o intermedio generando discapacidad, datos que concuerdan con Pérès y colaboradores, en su estudio cohorte Paquid, reportando una dependencia total física en el $20.7 \%$ de la población estudiada, un $42.9 \%$ con discapacidad leve y finalmente $29.5 \%$ con discapacidad moderada. ${ }^{34}$

Este aumento en la prevalencia puede ser explicado en primera instancia a la alta prevalencia de malnutrición y a la mala calidad de la dieta, además que pueden encontrarse discrepancias en el cribado de valoración utilizado para evaluar el desempeño físico en el estudio de Pérès y éste; en otros estudios, como el reporte INSERM, encontraron una discapacidad leve y moderada en el $40.4 \%$ y el $14 \%$ de la población respectivamente. ${ }^{35}$ La utilización de distintas herramientas de valoración puede generar variaciones en los resultados; por lo tanto, una evaluación de la fragilidad desde distintos ángulos puede desarrollar 
a futuro un consenso, una mejor definición y operacionalización del síndrome de fragilidad.

La movilidad, velocidad de la marcha y el equilibrio han demostrado su predictibilidad sobre el riesgo de sufrir caídas que pueden resultar en fracturas en el adulto mayor. Un reporte de Mulasso y colaboradores $^{36}$ sobre la movilidad y equilibrio, mostró un modelo predictivo sobre el riesgo de caídas, en el cual valora que las mujeres tienen 3.1 mayor riesgo de sufrir una caída en comparación con el hombre y 3.4 veces mayor riesgo de volver a tener un segundo evento en los siguientes 12 meses, en aquellas personas que sufrieron en el pasado una caída; por otro lado, también existe una relación estrecha de acuerdo a datos de Okochi y colaboradores ${ }^{37}$ con los cuatro indicadores relacionados con fragilidad como: velocidad de la marcha, aditamentos para caminar (bastón, andadera), deformación de espalda y el uso de medicamentos con el riesgo de sufrir caídas, por lo que las mujeres que presentan una movilidad pobre y alteración en la velocidad de la marcha o que hayan sufrido caídas en la vejez, se verá reflejado directamente sobre el desempeño físico y, por ende, en la aparición de fragilidad eventualmente, como en el caso de nuestra población. Por otro lado, actividades como levantarse de la silla, que son dependientes de fuerza muscular y potencia, son consideradas como una de las actividades diarias más demandantes muscularmente de acuerdo con estudios de Millor y colaboradores. ${ }^{38}$ En los resultados presentados se pudo corroborar esta aseveración, ya que el puntaje más bajo de las tres pruebas de desempeño físico fue obtenido en la prueba de levantarse de la silla. Este resultado puede ser explicado, en gran parte, a que son pruebas que comprometen diversas habilidades que debe llevar a cabo el cuerpo, tales como: movimiento del tronco, aceleración, velocidad, potencia e impulsos generados cuando se efectúa la tarea; por lo que, para poder mantener una estabilidad durante la moción del tronco, es necesaria una cantidad alta de energía y fuerza. ${ }^{39} \mathrm{En}$ cuanto a la aceleración y velocidad, Millor y colaboradores explican que se deriva directamente de la fuerza muscular $y$, finalmente, pueden existir irregularidades en los impulsos durante las fases de movimiento, lo cual puede explicar, de cierta manera, el puntaje obtenido de la población analizada; además, es probable que la alta prevalencia de malnutrición entre las mujeres estudiadas, sea causa de sarcopenia que repercute directamente en el desempeño funcional.

Finalmente, la asociación inversa entre el desempeño físico y el puntaje de la prueba de FRAIL coincide con lo propuesto por Corsonello y colaboradores; ${ }^{40}$ en su estudio sobre la significancia de la SPPB en el pronóstico de los pacientes adultos mayores encuentran, de igual manera, una relación inversa, denotando que la SPPB captura distintas dimensiones del desempeño físico, lo cual determinará y definirá las habilidades y el riesgo en la declinación de la funcionalidad física. No obstante, dentro de los resultados no se observó esta relación significativa, si se segmenta cada prueba de la batería de desempeño físico; las pruebas de equilibrio y levantarse de la silla no parecen mantener esta relación entre el desempeño físico y la presencia de fragilidad; probablemente, esto pudiera ser esclarecido debido al hecho de que la mayor parte de las participantes se encuentra en un estado de prefragilidad, por lo que el desarrollo o agravamiento de los síntomas de la fragilidad aún no han afectado en su totalidad; sin embargo, se han estudiado las manifestaciones iniciales del síndrome de fragilidad. Xue y colaboradores $^{41}$ describen la prevalencia de las afectaciones iniciales en las personas con prefragilidad en las cuales destacan: la debilidad (43.8\%), la lentitud, cansancio, baja de actividad física y pérdida de peso involuntaria $(22.8 \%)$; en dicho estudio se propone la 
debilidad (pérdida de fuerza) como principal detonante de la fragilidad, que genera una reacción en cadena en la aparición primaria de la lentitud y posteriormente del cansancio y pérdida de peso. La SPPB, al ser una prueba que evalúa principalmente la funcionalidad del tronco inferior, se estudió la alteración de la velocidad de la marcha como una de las primeras ma- nifestaciones clínicas afectadas de la prefragilidad. Parte de las limitaciones de este estudio, fue el seguimiento en la aplicación de la SPPB en diversos lapsos de tiempo, que pudiera evaluar la funcionalidad física a largo plazo, además de no poder conocer el nivel de sarcopenia y la calidad de la dieta de nuestras participantes.

\section{Conclusiones}

La valoración del síndrome de fragilidad, desde sus etapas iniciales, denota una importancia en la evolución de la vejez y permite identificar manifestaciones clínicas tempranas, por lo que es esencial la evaluación constante de los hábitos del adulto mayor, incluyendo aspectos físicos, mentales, sociales y dietéticos, por mencionar algunos. La fragilidad marcará el camino y desenlace del adulto mayor reflejado en su calidad de vida; las estrategias de prevención deben ser implementadas en la dieta y activación física individualizada en el adulto mayor. Finalmente, es fundamental la evaluación con diversas pruebas que puedan englobar la salud del adulto mayor que puedan apoyar a la definición concreta y específica del síndrome de fragilidad, que permita un mejor abordaje, retrasando el desarrollo de la aparición de manifestaciones clínicas y el tratamiento de este padecimiento.

\section{Conflicto de intereses}

Los autores declaran no tener ningún conflicto de intereses.

\section{Bibliografía}

1. Jin, K., Simpkins, JW, Ji, X., Leis, M. y Stambler, I. La necesidad crítica de promover la investigación del envejecimiento y las enfermedades relacionadas con el envejecimiento para mejorar la salud y la longevidad de la población de edad avanzada. Envejecimiento y enfermedad. 2015; 6(1):1.

2. Gutiérrez, R. y Lezana, F. Propuesta para un plan de acción en envejecimiento y salud. Serie: Cuadernillos de Salud Pública. México: Instituto Nacional de Geriatría. (2013).

3. González, G. y Rojas, A. Panorama demográfico en México: presente y futuro. Documento de trabajo. INGER. 2015.

4. Montaña, AM. Fragilidad y otros síndromes geriátricos. El residente. 2010; 5(2), 66-78.

5. Morley JE, Vellas B, Van Kan GA, Anker SD, Bauer $J M$, Bernabei $R$, et al. Frailty consensus: a call to action. J Am Med Dir Assoc. 2013; 14(6):392-7. doi: 10.1016/j.jamda.2013.03.022.

6. Kojima, G. Frailty as a predictor nursing home placement among community - dwelling older adults.
Journal of Geriatric Physical Therapy. 2018; 41(1) 42 -48.doi.org/10.1519/JTP.0000000000000097.

7. Menéndez, CR., Mauleón, LM., Condorhuaman, AP., y González, MJ. Aspectos terapéuticos de los grandes síndromes geriátricos. Tratamientos farmacológicos y no farmacológicos, indicaciones. Medicine - Programa de Formación Continua Acreditado. 2017;12 (46), 2743-2754.doi.org/10.1016/ j.m.2017.12.002

8. Clegg, A., Young, J., lliffe, S., Rikkert, M y Rockwood. Frailty in Eldery people. Lancet. 2013; 381, 752 - 752.doi.org/10.1016/S0140-6736(12)621679.

9. Johansen, LK., Delgado, C., Bao, Y y Kurella, TM. Frailty in dialysis initiation. Seminars in Dialysis. 2013; 26(6), 690-696.doi.org/10.1111/sdi.12126.

10. Fried, PL., Tangen, MC, Waltson, J., Newman, BA., Hirsch, C., Gottdiener, J., et al. Frailty in older adults; evidence for a phenotype. The Journal of Gerontology Series (a). 2001; 46, 146-156. doi.org/10.1093/ gerona/56.3.M146.

11. Rockwood, K y Mitnitski, A. Frailty in relation to the accumulation of deficits. The Journal of Gerontology 
Series 2007; 62,722-727. doi.org/10.1093/gerona/62.7.722

12. Woo J Leung J, Morley JE. Comparison of frailty indicators based on clinical phenotype and the multiple deficit approach in predicting mortality and physical limitation. Am Geriatr Soc. 2012; 60(8):1478-86. doi: 10.1111/j.1532-5415.2012.04074.x.

13. Areán García J, Suárez Linares $M$, López Llano $M L$, García García B, Suárez García FM. Frailty: A new challenge for physical medicine and rehabilitation specialists. Rehabilitación. 2017; 51(4):209-284.

14. Martínez, M., Córtez, G., Cartagena, P., Cano, C., Sánchez, L., y Leal, H. Assessment with Short Physical Performance Battery scale on functional ability in the eldery over 70 years. Atención Familiar. 2017; 24(4):145-149.

15. Treacy D, Hassett L. The Short Physical Performance Battery. J Physiother. 2018; 64(1):61. doi: 10.1016/j.jphys.2017.04.002.

16. Subra, J., Gillette, G., Cesari, M., Oustric, S y Vellas, B.The integration of frailty into clinical practice: Preliminary results from the Gérontopôle. The Journal of nutrition, health and aging. 2012; 16(8):714-720.

17. Bindawas, SM., Snih, AS., Ottenbacher, AJ., Graham, J., Protas, EE., Markides, KS., et al. Association between lower extremity performance and healthrelated quality of Life in elderly Mexican Americans. Journal of aging and health .2015; 27(6);10261045.doi.org/10.1177/0898264315572115.

18. Pritchard, JM., Kennedy, CC., Karampatos, S., Loannidis, G., Misiaszek, B., Marr, S., et al. Measuring frailty in clinical practice: a comparison of physical frailty assessment methods in a geriatric out - patient clinical. BMC Geriatrics.2017; 17(264), 1-8.doi. org/10.1186/s12877-017-0623-0.

19. Morley, JE., Malmstrom, TK y Miller, DK. A simple frailty questionnaire (FRAIL) predicts outcomes in middle aged African Americans. Journal Nutr Health Aging. 2012; 16(7):601-608.

20. Rosas, CO., Cruz, AE., Parra, RL., García, Gl., Contreras, GL y Szlejf, C. Cross -cultural and validation of the FRAIL scale to assess frailty in Mexican adults. Journal of the Medical Directors Association. 2016; 17(12), 1094-1098.doi.org/10.1016/j.jamda.2016.07.008.

21. Guralnik, JM., Simonsick, EM., Ferrucci, L., Glynn, RJ., Berkman, LF., Blazer, DG., et al. A short physical performance battery assessing lower extremity function: Association with self-reported disability and prediction of mortality and nursing home admission. The Journal of Gerontology.1994; 49(2), 8594.doi.org/10.1093/geronj/49.2.M85.

22. Bindawas, SM., Snih, AS., Ottenbacher, AJ., Graham, J., Protas, EE., Markides, KS., et al. Association between lower extremity performance and healthrelated quality of Life in elderly Mexican Americans. Journal of aging and health .2015; 27(6),10261045.doi.org/10.1177/0898264315572115.

23. Soler, P. A., Pavón, J. G., Lesende, I. M., \& Cortés, J. J. B. Detección y prevención de la fragilidad: una nueva perspectiva de prevención de la dependencia en las personas mayores. Medicina clínica, 2010;
135(15):713-719

24. Alcalá, M. V. C., Puime, Á. O., Santos, M. T. S., Barral, A. G., Montalvo, J. I. G., \& Zunzunegui, M. V. Prevalencia de fragilidad en una población urbana de mayores de 65 años y su relación con comorbilidad y discapacidad. Atención primaria, 2010; 42(10):520527.

25. Silguero, S. A. A., Martínez-Reig, M., Arnedo, L. G., Martínez, G. J., Rizos, L. R., \& Soler, P. A. Enfermedad crónica, mortalidad, discapacidad y pérdida de movilidad en ancianos españoles: estudio FRADEA. Revista Española de Geriatría y Gerontología, 2014; 49(2):51-58.

26. Martín-Lesende I, López-Torres JD, de-Hoyos MC, Baena JM, Gorroñogoitia A, Herreros Y. Detección e intervenciones en la persona mayor frágil en Atención Primaria. En: recomendaciones PAPPS (de la semFYC) 2014. Disponible en: http://www.papps. org/.

27. Bijlsma AY, Meskers CG, Van den Eshof N, Westendorp RG, Sipilä S, Stenroth L, Sillanpää $E$, et al. Diagnostic criteria for sarcopenia and physical performance Age (Dordr). 2014; 36(1):275-85.

28. Sillanpaa $E$, Stenroth $L$, Bijlsma $A Y$, Rantanen $T$, McPhee JS, Maden-Wilkinson TM, et al. Associations between muscle strength, spirometric pulmonary function and mobility in healthy older adults. Age. 2014; 36:9667. doi: 10.1007/s11357014-9667-7.

29. Piasecki $M$, Ireland $A$, Jones DA, McPhee JS. Agedependent motor unit remodelling in human limb muscles. Biogerontology. 2016; 17(3):485-496.

30. Vu HT, Nguyen TX, Nguyen TN, Nguyen AT, Cumming R, Hilmer $S$, et al. Prevalence of frailty and its associated factors in older hospitalised patients in Vietnam. BMC Geriatr. 2017; 17:216.

31. Walston JD. Sarcopenia in older adults. Curr Opin Rheumatol. 2012; 24:623-627. doi:10.1097/ BOR.0b013e328358d59b.

32. Bollwein J, Volkert D, Diekmann R et al. Nutritional status according to the mini nutritional assessment $(M N A(R))$ and frailty in community dwelling older persons: a close relationship. J Nutr Health Aging. 2013; 17:351-356. doi:10.1007/s12603-0130009-8.

33. Shikany JM, Barrett-Connor E, Ensrud KE et al. Macronutrients, diet quality, and frailty in older men. J Gerontol A Biol Sci Med Sci. 2014; 69:695-701. doi:10.1093/gerona/glt196.

34. Pérès K, Edjolo A, Dartigues JF, Barberger-Gateau $P$. Recent trends in disability-free life expectancy in the French elderly: twenty years follow-up of the Paquid cohort. Annual Review of Gerontology \& Geriatrics. 2013; 33:293-311. doi: 10.1891/01988794.33.293.

35. Inserm. Projet Dépendance 4 cohortes épidemiologiques: Haute Normandie, Paquid, 3 Cités et AMI. 2011 Noviembre 2011.

36. Mulasso, A., Roppolo, M., Gobbens, R. J., \& Rabaglietti, E. (2016). Mobility, balance and frailty in community-dwelling older adults: What is the best 1 -year predictor of falls? Geriatrics \& Gerontology 
International. doi:10.1111/ggi.12893.

37. Okochi J, Toba K, Takahashi T et al. Simple screening test for risk of falls in the elderly. Geriatr Gerontol Int. 2006; 6: 223-227.

38. Millor, N., Lecumberri, P., Gomez, M., Martinez, A., Martinikorena, J., Rodriguez-Manas, L., Izquierdo, M. Gait Velocity and Chair Sit-Stand-Sit Performance Improves Current Frailty-Status Identification. IEEE Transactions on Neural Systems and Rehabilitation Engineering. 2017; 25(11): 2018-2025. doi:10.1109/tnsre.2017.2699124.

39. Millor $\mathrm{N}$ et al., "Automatic evaluation of the 30-s chair stand test using inertial/magnetic-based technology in an older prefrail population," IEEE J. Bio- med. Health Inform. 2013; 14(4): 820-827.

40. Corsonello, A., Lattanzio, F., Pedone, C., Garasto, S., Laino, I., Bustacchini, S., et al. Prognostic Significance of the Short Physical Performance Battery in Older Patients Discharged from Acute Care Hospitals. Rejuvenation Research. 2012; 15(1), 41-48. doi:10.1089/rej.2011.1215.

41. Xue, Q.-L., Bandeen-Roche, K., Varadhan, R., Zhou, J., \& Fried, L. P. Initial Manifestations of Frailty Criteria and the Development of Frailty Phenotype in the Women's Health and Aging Study II. The Journals of Gerontology: Series A. 2008; 63(9), 984-990. doi:10.1093/gerona/63.9.984. 